\title{
Investigation of the presence of pregnancy rhinitis in the third trimester with rhinomanometry
}

\author{
Rustem Filiz ${ }^{1}$ Ahmet Ural ${ }^{1} \cdot$ Mehmet Ata Topcuoglu $^{2} \cdot$ Muharrem Dagli $^{3}$ \\ ${ }^{1}$ Department of Otorhinolaryngology, Faculty of Medicine, Bolu Abant Izzet Baysal University, Bolu, Turkey \\ ${ }^{2}$ Department of Obstetrics \& Gynecology, Faculty of Medicine, Bolu Abant Izzet Baysal University, Bolu, Turkey \\ ${ }^{3}$ Department of Otorhinolaryngology, Health Sciences University, Ankara Dişkapi Yıldırım Beyazıt Health Research \\ Hospital, Ankara, Turkey
}

\section{ABSTRACT}

\begin{abstract}
Aim: Pregnancy rhinitis (PR) is characterized with nasal symptoms during pregnancy without any signs of respiratory infection and it usually disappears within 2 weeks after delivery. We aimed to investigate the relationship between pregnancy rhinitis and findings derived from anterior rhinoscopy (AnR), anterior rhinomanometry (ARM) and subjective nasal obstruction score (SNOS).

Methods: This prospective, controlled study was performed in otorhinolaryngology and obstetrics and gynecology departments of our tertiary care center. A total of 30 pregnant women in the third trimester and 30 non-pregnant women were involved. All participants underwent otorhinolaryngology examination, as well as clinical evaluation for AnR, ARM and SNOS.

Results: Pregnancy rhinitis was detected in $66.7 \%$ of the pregnant women. The mean AnR was $3.60 \pm 1.35$ in pregnant women and $0.77 \pm 0.73$ in the control group. Total nasal inspiratory resistance (TNID) was $0.46 \pm 0.23$ in pregnant women and $0.27 \pm 0.06$ in the control group. The mean SNOS was $1.37 \pm 0.72$ in pregnant women and $0.57 \pm 0.63$ in the control group. AnR, ARM and SNOS findings were significantly higher in pregnant women $(p<0.05)$. There is a low positive and significant correlation between AnR, ARM, and SNOS values in pregnant women $(p<0.05)$.

Conclusion: Our data yielded that nasal obstruction and pregnancy rhinitis were common in pregnant women. Nasal symptoms and complaints must be carefully examined during pregnancy. Further prospective, controlled, randomized trials on larger series are warranted to elucidate the clinical and pathophysiological features of pregnancy rhinitis.
\end{abstract}

Keywords: Pregnancy rhinitis, subjective nasal obstruction score, anterior rhinoscopy, pregnancy rhinitis, anterior rhinomanometry.

$\triangle D r$. Ahmet Ural

Department of Otorhinolaryngology, Bolu Abant Izzet

Baysal University, Faculty of Medicine, Bolu, Turkey

E-mail: ahmetural@yandex.com.tr

Received: 2019-12-19 Revised: 2020-01-19

Accepted: 2020-02-13 / Published online: 2021-01-01

\section{Introduction}

Pregnancy rhinitis (PR) is defined as the presence of nasal symptoms occurring only during pregnancy for more than six weeks without any known allergic cause and no signs of respiratory infection. These symptoms disappear in the first two weeks after delivery. PR occurs in $20-30 \%$ of women between the 37 months of pregnancy $[1,2]$. The pathophysiological mechanism causing pregnancy rhinitis is that vasomotor imbalance in the nasal mucosa due to changes in estrogen 
and progesterone levels causes hyperemia and edema in the nasal mucosa. Also, placental growth hormone was found to be high in pregnancy rhinitis [3, 4].

Rhinitis does not directly affect pregnancy. However, uncontrolled rhinitis can increase stress by indirectly disturbing the nutritional and sleep status of the pregnant woman. Pregnancy hypertension, low Apgar score, preeclampsia and intrauterine growth retardation may be encountered due to rhinitis induced snoring $[5,6]$. Control of rhinitis during pregnancy reduces the risk of additional medication by increasing the quality of life of pregnant women $[7,8]$.

We aimed to investigate the frequency of pregnancy rhinitis and seek whether there is a correlation between anterior rhinoscopy (AnR) findings, anterior rhinomanometry (ARM) measurements, and subjective nasal obstruction scores (SNOS).

\section{Materials and Methods}

This prospective study was carried out by the otorhinolaryngology and obstetrics \& gynecology clinics of our institution. The study was approved by the local ethics committee and consent was obtained from all the participants (2017/73). A total of 30 pregnant women aged between 19-35 years who were in the third trimester of pregnancy were recruited. The control group included 30 non-pregnant women. The participant women had no history of allergic rhinitis (AR), septal deviation, and signs of acute or chronic upper respiratory tract infections, non-allergic rhinitis, nasal polyps, and sinonasal tumors.

\section{Outcome parameters}

A complete otorhinolaryngologic examination and decongestant-free active anterior rhinomanometry (ARM) were performed routinely for all participants.
All examinations and tests were performed between $27^{\text {th }}$ and $40^{\text {th }}$ weeks of pregnancy. In the history, pregnant women were asked to score the level of nasal congestion as for SNOS (none $=0$, mild $=1$, moderate $=2$, severe $=3$, fully blocked=4).

In the first examination, nulliparous women were asked whether they had PRs in the first and second trimesters. Multiparous women were asked whether they had PR in the previous pregnancies or during the current pregnancy. The gender of the baby was noted and pregnant women were asked to describe the nasal obstruction during pregnancy as either "increased", "decreased" or "unchanged".

In endoscopic examination, turbinate hypertrophy in a nasal cavity was scored by 1 point, the mild congestion of the mucosa was scored as 1 point and severe congestion was scored as 2-points. Therefore, the highest score a pregnant can get in anterior rhinoscopic examination was 6 points [9].

Decongestant-free ARM was performed on all pregnant women by using Rhinostream SRE 2000 (Interacoustics A/S, Drejervaenget 8, DK5610 Assens, and Denmark) rhinomanometry device. The mask covered both the mouth and the nose. The pressure probe passed through the mask was placed in a nostril and the nasal flow probe in the other nostril. During placement of the probes, attention was paid not to deform the nostrils and to prevent the air leakage. Pregnant women were asked to keep their mouths closed and breathe through their noses. The values were read at $150 \mathrm{~Pa}$. After expiration and inspiration, nasal resistance was recorded in $\mathrm{Pa} / \mathrm{cm}^{3}$ and the individual resistances of each nostril and the total inspiratory nasal resistance were calculated. Patients who had nose clearance before ARM had rested for 20 minutes in a room with a temperature of $20 \pm 3$ ${ }^{\circ} \mathrm{C}$, the humidity was $50 \%$, and the illumination 
was moderate. The patients were instructed about the test. They had not exercised for 2 hours preceding the test and they were asked not to have tea, coffee, and cigarettes.

Results from the pregnant group were compared with 30 non-pregnant women in the control group who were non-smokers and did not have any signs of acute upper respiratory tract infections, or chronic sinonasal diseases. Thus, AnR, ARM and SNOS scores were recorded under same conditions.

\section{Statistical analysis}

Statistical analysis was performed by using SPSS 24.0 software (Statistical Package for Social Sciences Inc., Chicago, IL, USA). In terms of descriptive statistical methods, Mann Whitney $U$ test for difference tests and Spearman Brown test for correlation were used since the data displayed non-parametric features. The results were evaluated with a confidence interval of $95 \%$ and a p-value less than 0.05 was considered as statistically significant.

\section{Results}

The mean ages in the study and control groups were $30.43 \pm 3.65$ and $30.37 \pm 4.07$, respectively. The gestational age during examination ranged between 27 and 39 weeks (mean: 34.2 \pm 3.71 ; median: 36). Of the 30 pregnant women, 9 $(30 \%)$ were nulliparous and $21(70 \%)$ were multiparous. A total of 16 newborns were male $(53.3 \%)$ and 14 (46.7\%) were female.

None of the pregnant women reported any relief of nasal congestion during pregnancy. The complaints did not change in 11 women $(36.7 \%)$ and were increased in 19 women $(63.3 \%)$. Fifteen of 21 multiparous women (71.4\%) reported absence of PR, while 6 (28.6\%) of them reported presence of PR in their previous pregnancies. In their current pregnancies, no PR were diagnosed in 10 pregnant women (33.3\%), while PR was diagnosed in 20 pregnant women $(66.7 \%)$ (Table 1).

Table 1. Gestational features and rhinitis findings in pregnant women.

\begin{tabular}{|l|l|}
\hline Parameters & $\begin{array}{l}\text { Study group } \\
(\mathbf{n}=30)\end{array}$ \\
\hline Average pregnancy weeks & $34.2 \pm 3.71$ \\
\hline Median pregnancy weeks & 36 \\
\hline Nulliparous & $30 \%(n=9)$ \\
\hline Multiparous & $70 \%(n=21)$ \\
\hline Nasal congestion decreased & $0 \%(n=0)$ \\
\hline Nasal congestion unchanged & $36.7 \%(n=11)$ \\
\hline Nasal congestion increased & $63.3 \%(n=19)$ \\
\hline Presence of rhinitis & $66.7 \%(n=20)$ \\
\hline Absence of rhinitis & $33.3 \%(n=10)$ \\
\hline
\end{tabular}

The mean ANR values in the study and control groups were $3.60 \pm 1.35$ and $0.77 \pm 0.73$, respectively. Therefore, pregnant women exhibited higher AnR scores compared to nonpregnant women.

Regarding ARM findings, the mean total nasal inspiratory resistance (TNID) was $0.46 \pm 0.23$ in the study group and $0.27 \pm 0.06$ in the control group. The minimum and mode TNID scores were similar between 2 groups ( 0.19 and 0.18 ; 0.24 and 0.23 ). However, the maximum TNID was 0.39 in the control group and 1.07 in the study group. Therefore, the average TNID score was higher in pregnant women. Moreover, TNID values were grouped according to 0.12 $0.33 \mathrm{~Pa} / \mathrm{ml} / \mathrm{s}$, which was accepted as the normal value range in rhinomanometric measurements. With reference to this threshold, $9(30 \%)$ of pregnant women in the study group and 25 (83.3\%) of the non-pregnant women in the control group were within this range. 
Table 2. AnR, ARM and SNOS results in the study and control groups.

\begin{tabular}{|l|l|l|l|l|l|}
\hline Groups & Minimum & Maximum & Average & Median & Mode \\
\hline $\begin{array}{c}\text { Study group } \\
\text { AnR } \\
\text { ARM } \\
\text { SNOS }\end{array}$ & 0 & 6 & $3.60 \pm 1.35$ & 4 & 4 \\
\cline { 2 - 6 } & 0.19 & 1.07 & $0.46 \pm 0.23$ & 0.39 & 0.24 \\
\cline { 2 - 6 } $\begin{array}{c}\text { Control group } \\
\text { AnR }\end{array}$ & 0 -none & 3 -severe & $1.37 \pm 0.72$ & 1 -mild & 1 -mild \\
\hline ARM & 0.18 & 2 & $0.77 \pm 0.73$ & 1 & 1 \\
\hline SNOS & 0 -none & 2 -moderate & $0.57 \pm 0.63$ & 0.50 -mild & 0 -none \\
\hline
\end{tabular}

[Abbreviations: *Anterior rhinoscopy (AnR), anterior rhinomanometry (ARM) and subjective nasal obstruction score (SNOS)].

Table 3. Comparison of AnR, ARM and SNOS results in the study and control groups.

\begin{tabular}{|c|c|c|c|c|c|c|c|}
\hline & $\begin{array}{l}\text { Groups } \\
(n=30)\end{array}$ & $\bar{x}$ & Ave. Row & Rows Total & $\mathbf{U}$ & $\mathbf{Z}$ & p-value* \\
\hline \multirow{2}{*}{ AnR } & Study & 3.60 & 44.20 & 1326.00 & \multirow{2}{*}{39.000} & \multirow{2}{*}{-6.200} & \multirow{2}{*}{$<0.00$} \\
\hline & Control & 0.77 & 16.80 & 504.00 & & & \\
\hline \multirow{2}{*}{ ARM } & Study & 0.46 & 40 & 1200.00 & \multirow{2}{*}{165.000} & \multirow{2}{*}{-4.219} & \multirow{2}{*}{$<0.00$} \\
\hline & Control & 0.27 & 21 & 630.00 & & & \\
\hline \multirow{2}{*}{ SNOS } & Study & 1.37 & 38.88 & 1166.50 & \multirow{2}{*}{198.500} & \multirow{2}{*}{-4.042} & \multirow{2}{*}{$<0.00$} \\
\hline & Control & 0.57 & 22.12 & 663.50 & & & \\
\hline
\end{tabular}

* Mann-Whitney U Test. [Abbreviations: **Anterior rhinoscopy (AnR), anterior rhinomanometry (ARM) and subjective nasal obstruction score (SNOS)].

On the other hand, the mean SNOS in the study and control groups were $1.37 \pm 0.72$ and $0.57 \pm 0.63$, respectively. We noted that SNOS averages of pregnant women were significantly higher than non-pregnant women (Table 2). The analysis of descriptive findings yielded that AnR, ARM and SNOS displayed significant differences between study and control groups $(p<0.05)$. AnR, ARM and SNOS findings of in pregnant women were significantly higher than the control group (Table 3).
In the study group, the correlation between AnR, ARM and SNOS was sought and the correlation between AnR and ARM was 0.176. The correlation between AnR and SNOS was 0.295 and the correlation between ARM and SNOS was 0.197. These correlations were found to be low and positive, but they were significant $(p<0.05)$.

As for these results, if any of AnR, ARM and SNOS values of the pregnant women increased, the others also tended to increase mildly. The 
determination coefficient (explained variance $=r^{2}$ ) was calculated to determine the impact of changes of AnR, ARM and SNOS measurements on the other. As a result, $\mathrm{r}^{2}$ value between AnR and ARM was found to be 3.1. This value was 8.7 between AnR and SNOS; and it was 3.8 between ARM and SNOS. Any changes in AnR or ARM constitutes an alteration of $3.1 \%$. In the other parameters, these percentages were found to be $8.7 \%$ and $3.8 \%$.

Furthermore, the regression equation between AnR and SNOS was $y=0.6+0.21 x$. It was $\mathrm{y}=1.16+0.46 \mathrm{x}$ between ARM and SNOS, and $y=0.39+0.02 x$ between AnR and ARM.

In the control group, the correlation was 0.134 between AnR and ARM, 0.131 between AnR and SNOS and -0.36 between ARM and SNOS. Therefore, the correlations between AnR-ARM and AnR-SNOS were low and positive. However, the relationship between ARM and SNOS was very low and negative.

As for these results, $\mathrm{r}^{2}$ between AnR and ARM was $1.8, \mathrm{r}^{2}$ between AnR and SNOS was 1.7, and $\mathrm{r}^{2}$ between ARM and SNOS was 0.1. These findings may be interpreted as any change in AnR or ARM constitutes $1.8 \%$ change. In the other parameters, these percentages were $1.7 \%$ and $0.1 \%$.

\section{Discussion}

In the present study, the prevalence of PR was $66.7 \%$ and it was reported as $40 \%$ in previous publications [10]. The nasal obstruction symptom evaluation (NOSE) scores in the study and control groups displayed no significant differences $(p=0.866)$. Minimal cross sectional areas at internal nasal valve and at the level of the head of inferior turbinate decreased significantly between the first and third trimesters (2). There was no difference between the trimesters as for total nasal resistance. The correlation analysis between the NOSE score, total volume and minimal cross sectional area at internal nasal valve demonstrated no significant differences [11].

In Sweden, the prevalence of PR was found to be $22 \%$ in a series of 599 patients [12]. Thus, it is possible to say that the global prevalence is considerably higher than the prevalence in Turkey. However, relatively larger samples should be studied for achievement of more objective outcomes.

We noted that nasal obstruction and PR were common during pregnancy. The previous PR was found in $28.6 \%$ of multiparous pregnancies. The presence of rhinitis in the current pregnancy was detected as $66.7 \%$. The mean AnR score was $3.60 \pm 1.35$. Our results were close to the value of 3.47 reported by Philpott et al. [13]. The utilities of AnR and ARM has been recommended for evaluation of rhinitis and nasal physiological changes during pregnancy [13]. Subjective nasal obstruction scale (SNOS) has been validated as a reliable tool of nasal congestion in previous studies [14].

Ülkümen et al. [15] reported that an incidence of $38.89 \%$ for PR. Nasal congestion was significantly associated with body-mass index and gestational weeks. Patients should be informed about adverse fetal and maternal outcomes of pregnancy related nasal congestion which may be aggravated by obesity and excessive weight gain during pregnancy.

In our study, the mean ARM was $0.46 \pm 0.23$ and the mean SNOS was $1.37 \pm 0.72$. The correlations between AnR-ARM, ARM-SNOS and AnR-SNOS were 0.176, 0.195 and 0.295, respectively.

The nasal obstruction associated with PR may lead to with diminution of the quality of life and sleep [16]. Gilbey et al. [17] suggested that 
rhinosinusitis-specific quality of life was lower in the third trimester compared to that of the second trimester and that of patients without rhinosinusitis. Thus, increased awareness is important to improve the quality of life in pregnant women, prevent sleep apnea and related morbidity, and positively affect the gestational outcomes. There is controversy on the influence of atopy on the pregnancy outcomes since some authors postulated that atopy may be associated with more favorable results [17]; whereas some publications indicated that there was no link between atopy and recurrent abortions [18,19].

The main limitations of our study include relatively small sample size, subjectivity of detection of SNOS and data limited to the experience of a single center. On the other hand, prospective design and integrity of specific data constituted the strengths of our study.

\section{Conclusions}

To conclude, nasal obstruction and PR are common conditions in pregnant women. As reflected in higher scores of AnR, ARM and SNOS values, nasal symptoms and complaints must be questioned during follow-up in pregnancy. A low positive, but significant correlation was noted between AnR, ARM and SNOS measurements in pregnant women. The implementation of further multicentric trials on larger series may aid in accomplishment of more accurate results.

Funding: There is no financial support and sponsorship

Conflict of Interest: The authors declare that they have no conflict of interest.

Ethical statement: The study was approved by Bolu Abant Izzet Baysal University local ethics committee (Decision number: 2017/73).

\section{ORCID iD of the author(s)}

Rustem Filiz / 0000-0003-4778-3498

Ahmet Ural / 0000-0002-6088-1415

Mehmet A Topcuoglu / 0000-0002-8929-6973

Muharrem Dagli / 0000-0003-2099-9395

Copyrights: (C)2021@author (s).

This is an open access article distributed under the terms of the Creative Commons Attribution License (CC BY 4.0), which permits unrestricted use, distribution, and reproduction in any medium, provided the original author(s) and source are credited and that the original publication in this journal is cited, in accordance with accepted academic practice. No use, distribution or reproduction is permitted which does not comply with these terms.

\section{References}

[1]Caparroz FA, Gregorio LL, Bongiovanni G, et al. Rhinitis and pregnancy: literature review. Braz J Otorhinolaryngol. 2016; 82(1):105-11.

[2]Singla P, Gupta M, Matreja PS, et al. Otorhinolaryngological complaints in pregnancy: a prospective study in a tertiary care centre. Int J Otorhinolaryngol Head Neck Surg. 2015; 1(2):75-80.

[3]Källén, BAJ, Olausson PO. Use of oral decongestants during pregnancy and delivery outcome. Am J Obstet Gynecol. 2006; 194(2):480-85.

[4]Ellegård E, Oscarsson J, Bougoussa $\mathrm{M}$, et al. Serum level of placental growth hormone is raised in pregnancy rhinitis. Arch Otolaryngol Head Neck Surg. 1998; 124(4):439-43.

[5]Ayrım A, Keskin EA, Ozol D, et al. Influence of self-reported snoring and witnessed sleep apnea on gestational hypertension and fetal outcome in 
pregnancy. Arch Gynecol Obstet. 2011; 283(2):195-99.

[6]Franklin KA, Holmgren PÅ, Jönsson F, et al. Snoring pregnancy-induced hypertension and growth retardation of the fetus. Chest. 2000; 117(1):137-41.

[7]Rundcrantz H. Postural variations of nasal patency. Acta Oto-Laryngologica. 1969; 68(5):435-43.

[8]Ellegård EK. Pregnancy rhinitis. Immunol Allergy Clin N Am. 2006; 26(1):119-35.

[9]Priftis KN, Drigopoulos K, Sakalidou A, et al. Subjective and objective nasal obstruction assessment in children with chronic rhinitis. Int J Ped Otorhinolaryngol. 2006; 70(3):501-5.

[10]Bende M, Gredmark T. Nasal stuffiness during pregnancy. Laryngoscope. 1999; 109(7 Pt 1):1108-10.

[11]Demir UL, Demir BC, Oztosun E, et al. The effects of pregnancy on nasal physiology. Int Forum Allergy Rhinol. 2015; 5(2):162-66.

[12] Ellegård E, Karlsson G. Nasal congestion during pregnancy. Clin Otolaryngol. 1999; 24(4):307-11.

[13]Philpott CM, Conboy P, Al-Azzawi F, et al. Nasal physiological changes during pregnancy. Clin Otolaryngol. 2004; 29(4):343-51.

[14]Pelen A, Tekin M, Özbilen Acar G, et al. Comparison of the effects of radiofrequency ablation and microdebrider reduction on nasal physiology in lower turbinate surgery. Kulak Burun Bogaz Ihtis Derg. 2016; 26(6):325-32.

[15]Ulkumen B, Ulkumen BA, Pala HG, et al. Pregnancy rhinitis in Turkish women: Do gestational week, BMI and parity affect nasal congestion? Pak J Med Sci. 2016; 32(4):950-54.
[16] Namazy JA, Schatz M. Asthma and rhinitis during pregnancy. Mt Sinai J Med. 2011; 78(5):661-70.

[17] Gilbey P, McGruthers L, Morency AM, et al. Rhinosinusitis-related quality of life during pregnancy. Am J Rhinol Allergy. 2012; 26(4):283-86.

[18] Savilahti E, Siltanen M, Pekkanen J, et al. Mothers of very low birth weight infants have less atopy than mothers of fullterm infants. Clin Exp Allergy. 2004; 34(12):1851-54.

[19]Mattar R, Camano L, Daher S. Recurrent spontaneous abortion and atopy. Rev Bras Ginecol Obstet. 2003; 25(5):331-35. 